\title{
Pratiques
}

Linguistique, littérature, didactique

$173-174 \mid 2017$

Le déjà-là dans l'écriture

\section{Reprise, reformulation et réappropriation : les traces de discours antérieurs dans les slogans écrits du printemps érable}

Repeat, reformulation and reappropriation: the traces of previous discourses in the written slogans from Quebec's 2012 student strike

\section{Geneviève Bernard Barbeau}

\section{OpenEdition}

Journals

Édition électronique

URL : http://journals.openedition.org/pratiques/3258

DOI : $10.4000 /$ pratiques.3258

ISSN : 2425-2042

Éditeur

Centre de recherche sur les médiations (CREM)

Référence électronique

Geneviève Bernard Barbeau, «Reprise, reformulation et réappropriation : les traces de discours

antérieurs dans les slogans écrits du printemps érable », Pratiques [En ligne], 173-174 | 2017, mis en ligne le 10 mars 2016, consulté le 20 avril 2019. URL : http://journals.openedition.org/pratiques/3258 ; DOI : 10.4000/pratiques.3258

Ce document a été généré automatiquement le 20 avril 2019

(c) Tous droits réservés 


\title{
Reprise, reformulation et réappropriation : les traces de discours antérieurs dans les slogans écrits du printemps érable
}

\author{
Repeat, reformulation and reappropriation: the traces of previous discourses in \\ the written slogans from Quebec's 2012 student strike
}

Geneviève Bernard Barbeau

1 En 2012, le Québec a connu la plus importante grève étudiante de son histoire, causée par la décision du gouvernement de l'époque, formé par le Parti libéral avec à sa tête le premier ministre J. Charest, de procéder à une hausse des frais de scolarité universitaires de $1625 \$$ sur cinq ans, une augmentation de $75 \%$ par rapport au tarif alors existant. Sans surprise, une importante opposition s'est fait entendre dans le milieu associatif étudiant avant de s'étendre à une large tranche de la population québécoise. De là a été déclenchée, en février, une grève ayant pour but premier l'annulation de la hausse des frais de scolarité annoncée, bien que de nombreuses autres revendications aient été au cœur du mouvement : gel des frais, gratuité scolaire, etc. Plus de 300000 étudiants sur les 400000 que comptait alors la province ont pris part à cette grève, qui a duré près de sept mois et qui a assurément constitué l'évènement le plus marquant de l'année. Les manifestations qui l'ont caractérisée, dont l'ampleur n'avait encore jamais eu d'égale, ont été marquées par un affrontement parfois violent avec les forces policières et ont divisé la population, laissant entrevoir de vives tensions sociales ${ }^{2}$. La grève étudiante a d'ailleurs rapidement été qualifiée de printemps érable, en référence au printemps arabe de 2011 et en clin d'œil au sirop d'érable québécois.

2 Parmi la production discursive découlant de ce vaste mouvement de contestation, les slogans entendus ou vus lors des manifestations ont suscité l'intérêt dans l'espace public, notamment en raison de leur caractère jugé tantôt choquant (gros mots, jurons, insultes), tantôt inventif (jeux de mots, références culturelles, etc.). C'est à cet objet, et plus 
particulièrement au slogan revendicateur sous sa forme scripturale, c'est-à-dire rédigé sur des pancartes brandies lors de manifestations pro-étudiantes, que cet article est consacré. Nous nous intéressons plus spécifiquement à la façon dont les manifestants ont recours au discours d'autrui - ce « déjà-là » qui est au cœur du processus d'écriture pour construire leurs propres énoncés revendicateurs.

\section{Slogans et discours en circulation}

Le slogan est une «formule concise et frappante, facilement repérable, polémique et le plus souvent anonyme, destinée à faire agir les masses » (Reboul, 1975, p. 42). Il s'agit généralement d'une simplification et d'un résumé d'idées, de situations ou d'idéologies complexes afin que l'accent soit mis sur un de leurs aspects particuliers et que l'attention y soit attirée (Denton, 1980). Dans le cas des slogans revendicateurs, l'objectif est de faire état d'une émotion "contre ", d'appeler à la mobilisation et de faire pression sur son opposant, le tout de manière à marquer l'esprit, tant par le fond que par la forme du message.

Parmi les éléments caractéristiques des slogans écrits du printemps érable, on note, sans surprise, leur inscription dans un vaste réseau de discours préalablement énoncés. M. Bakhtine (1929) l'affirmait il y a déjà près d'un siècle, tout discours ou presque est traversé de propos antérieurs qui sont repris et transformés par les locuteurs, que ce soit consciemment ou non. Ainsi, lorsqu'un individu prend la parole, il fait constamment référence aux dires d'autrui ou à ses propres dires, et leur reprise participe à la construction de la mémoire discursive d'un groupe. On parle alors de circulation des discours, phénomène qui «englobe des mécanismes d'appropriation (déclencheurs de circulation), de réalisation (c'est-à-dire de réénonciation) et de remises en relation discursives (propagateurs) relativement organisés entre des espaces discursifs (textes, genres de textes, formations discursives) par des agents de circulation» (Rosier, 2003, p. 69). Or, « faire circuler des propos, c'est leur donner une seconde ou une troisième vie ; c'est leur offrir d'autres voix pour se faire entendre, c'est aussi leur donner de l'importance en leur accordant une certaine légitimité » (Vincent, Laforest \& Turbide, 2007, p. 197). Autrement dit, la réactivation en discours de référents et de lieux communs à un groupe permet leur inscription dans la mémoire collective.

5 Si ce phénomène est loin d'être propre aux slogans écrits du printemps érable, il n'en demeure pas moins que certains mécanismes de circulation, eux, le sont. Par ailleurs, si les slogans de la grève étudiante québécoise ont retenu l'attention jusqu'à maintenant, c'est surtout en raison, au plan lexical, du vocabulaire employé (Vincent, 2014) ou, au plan sociodiscursif, de la manière dont ils constituent une forme d'appel à l'action originale et efficace (Bernard Barbeau, 2015). Aucune attention n'a été portée à la façon dont les manifestants ont utilisé le matériau discursif à leur disposition pour construire leur propre discours revendicateur au moyen de la forme brève qu'est le slogan écrit. C'est ainsi que, nous inscrivant dans une approche sociodiscursive de l'analyse de la circulation des discours (Rosier, 2003 ; López Muños, Marnette \& Rosier, 2004 ; Turbide, Vincent \& Laforest, 2010), nous nous proposons d'étudier la reprise, la réinterprétation et la remise en discours des propos d'autrui dans les slogans scripturaux du printemps érable. Il s'agira plus spécifiquement de faire ressortir la manière dont les individus réactualisent et remettent en circulation des discours du passé - proche ou lointain pour appuyer leur point de vue. 


\section{Cadre théorique et méthodologique}

Notre analyse est fondée sur un corpus ${ }^{3}$ de 780 slogans rédigés sur des pancartes lors de manifestations pro-étudiantes qui ont eu lieu au plus fort de la grève. Plusieurs banques de photos des manifestations étudiantes existent en ligne (blogues, pages Facebook et Tumblr consacrées au printemps érable, etc.), et c'est à partir de celles-ci que le corpus non exhaustif compte tenu de la quantité innombrable de slogans ayant circulé pendant la grève, mais néanmoins suffisamment étendu pour en observer les régularités - a été constitué.

7 Partant de ce vaste ensemble de données, nous nous sommes inspirée d'un modèle d'analyse, proposé par O. Turbide, D. Vincent et M. Laforest (2010) et présenté ci-dessous, qui recense les principaux facteurs qui entrent en jeu lorsqu'un discours est en circulation.

Tableau 1. Modèle d'analyse de la circulation des discours

\begin{tabular}{|c|c|}
\hline \multicolumn{2}{|c|}{ 1. Contexte de production du discours source et de la reprise } \\
\hline $\begin{array}{l}\text { a) Auteur des propos sources et de } \\
\text { l'agent de circulation }\end{array}$ & $\begin{array}{l}\text { Caractéristiques socioprofessionnelles de l'auteur des propos sources et de } \\
\text { l'agent de circulation; rôle social; données personnelles, etc. }\end{array}$ \\
\hline b) Type de propos en circulation & $\begin{array}{l}\text { Forme : mot, expression, énoncé, action discursive, texte, événement } \\
\text { Contenu : information factuelle, concepts, idées, propos } \\
\text { valorisant/dévalorisant autrui }\end{array}$ \\
\hline c) Voies de circulation & $\begin{array}{l}\text { Voie de circulation privée, publique ou médiatique ; circonstances dans } \\
\text { lesquelles les propos sources et les reproductions ont été produits }\end{array}$ \\
\hline \multicolumn{2}{|c|}{ 2. Rapport entre le discours source et la reprise } \\
\hline $\begin{array}{l}\begin{array}{l}\text { a) Maillon de la chaîne de } \\
\text { circulation }\end{array} \\
\end{array}$ & Reprise du discours source, reprise d'une reprise, brouillage du lieu d'emprunt \\
\hline b) Types formels de reprise & $\begin{array}{l}\text { Exhibée } \\
\text { (Marques explicites de discours rapporté) }\end{array}$ \\
\hline $\begin{array}{l}\text { c) Degré de conformité de la } \\
\text { reprise avec la source }\end{array}$ & $\longrightarrow$ Transformation profonde \\
\hline $\begin{array}{l}\text { d) Tonalité de la source et de la } \\
\text { reprise }\end{array}$ & Dysphorique ou euphorique \\
\hline $\begin{array}{l}\text { e) Orientation de la reprise avec les } \\
\text { propos sources }\end{array}$ & Co-orientée (convergente) ou anti-orientée (divergente) \\
\hline
\end{tabular}

8 Ce modèle, qui combine des éléments de description linguistique, discursive et sociopragmatique, repose sur l'idée que tout acte de langage produit un effet sur les participants à la situation de communication. Au-delà d'une caractérisation purement linguistique des discours en circulation, l'ensemble des paramètres permet de tenir compte du positionnement des agents de circulation sur les idées qu'ils propagent, ce qui inscrit directement ce modèle dans une approche résolument sociodiscursive de l'analyse de la circulation des discours.

9 Des différents éléments consignés dans ce modèle d'analyse, nous nous intéressons principalement au rapport entre le discours source et la reprise. Nous n'excluons pas le contexte de production du discours source et de la reprise, auquel nous ferons allusion lors de l'analyse. Toutefois, en raison du corpus, constitué de slogans brandis par des manifestants souvent anonymes, il nous est difficile de rendre compte de façon systématique des caractéristiques des agents de circulation et, parfois, de l'auteur des propos sources - cela est particulièrement vrai lors de la reprise de slogans « classiques " que l'on observe dans presque toutes les manifestations et qui ne sont pas toujours 
attribuables à un individu propre. En ce qui a trait aux voies de circulation, nous nous intéressons ici à un seul cas, la reprise de discours antérieurs dans des slogans rédigés sur des pancartes lors de manifestations qui ont lieu dans l'espace public. La prise en compte de ce paramètre n'est donc pas nécessaire dans le cadre de notre analyse. Il est toutefois pertinent d'identifier le type de propos en circulation : tantôt d'autres slogans, tantôt des mots-clés, des portions de phrases ou de discours entiers, tantôt des évènements autres dont la part discursive est plus ou moins grande ; nous le préciserons au fur et à mesure de l'analyse.

Suivant les paramètres d'analyse sélectionnés, nous avons identifié trois modèles prototypiques de la remise en discours de propos antérieurs dans les slogans du printemps érable. Autrement dit, parmi les slogans analysés, ceux qui contiennent des traces de discours préalablement énoncés s'articulent en trois catégories: la reprise d'autres discours revendicateurs, celle de discours de l'opposant et celle de références culturelles qui, de prime abord, n'entretiennent aucun lien avec les revendications étudiantes. Chaque catégorie possède des caractéristiques qui lui sont propres et est construite selon des mécanismes sociodiscursifs particuliers. Davantage qu'un relevé de l'ensemble des slogans lui appartenant, c'est la façon dont chaque prototype est construit qui sera examinée, ce qui fera apparaitre la manière dont est employé, réemployé ou réinvesti le « déjà-là » dans l'écriture revendicatrice populaire.

\section{La reprise de discours revendicateurs}

11 De mai 68 aux manifestations pour le mariage pour tous en passant par le printemps érable, certains slogans constituent des classiques que l'on observe dans la quasi-totalité des rassemblements contestataires, quelle que soit la cause revendiquée. Sans surprise, on trouve aussi plusieurs de ces slogans - les «slogans prototypiques " pour reprendre l'expression de Y. Grinshpun (2013) - dans notre corpus. On constate alors l'importance qu'ils revêtent dans l'imaginaire protestataire, et le fait d'y avoir recours permet aux manifestants de s'inscrire dans un mouvement de contestation en apparence sans frontières, ni géographiques ni temporelles. "Quand l'injustice devient la loi, la résistance est un devoir ", "Sous les pavés, la grève » et "Le peuple uni jamais ne sera vaincu » (El pueblo unido jamás será vencido) constituent à n'en pas douter de ces slogans utilisés au point tel qu'ils peuvent être considérés comme des stéréotypes, voire des clichés, de la parole protestataire.

Si les deux premiers slogans sont inscrits sur plusieurs pancartes dans notre corpus, ils constituent chaque fois une reprise intégrale, c'est-à-dire qu'ils sont en parfaite conformité avec le slogan source, qui ne subit aucune transformation. Il y a donc peu à dire sur les modalités de leur remise en discours. Le troisième slogan, en revanche, est plus intéressant pour rendre compte des mécanismes de circulation : la phrase a souvent été légèrement modifiée pour se lire ainsi « Un peuple instruit jamais ne sera vaincu ». Dans un tel cas, la reprise constitue une transformation mineure qui vise, par l'adjectif instruit, à inscrire directement le slogan au cœur des revendications étudiantes, qui concernent l'accès à l'éducation. Sur le plan formel, les marques de reprise sont presque toujours effacées - on ne fait pas référence à l'auteur et on n'utilise pas de guillemets pour marquer la présence de discours rapporté, ce qui est également le cas des autres slogans appartenant à cette catégorie. Une pancarte est néanmoins particulièrement intéressante dans la mesure où le slogan, rédigé dans sa version originale en espagnol, ce 
qui constitue une reprise intégrale, est accompagné d'un portrait d'E. Guevara dont le visage a été remplacé par celui de R. Lévesque, premier ministre du Québec de 1976 à 1985 et très étroitement associé à l'émancipation des Québécois francophones et à la construction d'une identité québécoise forte. Cela conduit, par une certaine forme d'exhibition de la reprise, à une association entre ce qui est présenté comme l'auteur de la citation originale - ce qui n'est toutefois pas exact - et un politicien emblématique du Québec moderne. En effet, bien que les marques de discours rapporté soient absentes, le portrait d'E. Guevara joue ici en quelque sorte le rôle de signature, donnant au slogan une apparence de véritable citation marquée comme telle. Cette confusion concernant le premier énonciateur de cette phrase aujourd'hui mythique doit être soulignée. Or, c'est surtout le lien étroit entre un personnage révolutionnaire connu, E. Guevara, des paroles revendicatrices qui sont (à tort) faites siennes et une figure politique québécoise associée à la volonté de changement et dont les idéaux politiques étaient à l'opposé de ceux du Parti libéral qui est révélateur. Tout en usant d'une tonalité euphorique et d'une coorientation avec le slogan source, les manifestants font montre d'une originalité qui témoigne de leur imaginaire revendicateur : E. Guevara et R. Lévesque, chacun porteur, bien que de façon différente, d'une certaine révolution, sont ici présentés comme emblématiques du mouvement étudiant, qui se voit dès lors revêtir un caractère plus grand que nature. Le combat dépasse la question de la hausse des frais de scolarité ; il s'agit d'un réel bouleversement qui fait appel à certains des pères de la révolution et du changement social.

\section{La réappropriation et le détournement du discours de l'opposant}

La circulation des discours ne concerne pas uniquement la citation plus ou moins fidèle de propos sources pour appuyer un point de vue allant dans le même sens que le sien; il est également possible de transformer le discours ou les idées de son opposant et de se les réapproprier à des fins de revendication. C'est ainsi que, dans le corpus analysé, on observe une quantité importante de slogans formés à partir du détournement des propos d'individus opposés aux revendications étudiantes, à plus forte raison des membres du gouvernement. Évidemment, ces slogans s'inscrivent de plain-pied dans une forme de discours « contre ", où la tonalité dysphorique et l'antiorientation avec les propos sources sont nettement identifiables.

Par exemple, plusieurs écrits font référence à une loi promulguée expressément pendant le conflit étudiant afin d'encadrer étroitement (ou de restreindre, selon plusieurs) les manifestations. Cette loi oblige notamment les manifestants à remettre le plan de leur trajet aux forces policières, sans quoi le rassemblement est décrété illégal et les individus qui y prennent part courent le risque de se faire arrêter. C'est dans ce contexte qu'émergent des écrits de la même nature que celui-ci : "Où s'en va le Québec? M. Charest, je veux connaitre votre itinéraire ", où la référence à l'itinéraire est double. Il s'agit d'une part d'un rappel de la loi promulguée par le gouvernement Charest et, de l'autre, d'une critique non seulement de cette nouvelle mesure, mais également de l'ensemble des politiques mises en place par le gouvernement, présentées comme floues ou peu cohérentes. Nous en convenons, il s'agit moins de la remise en discours de propos sources que de l'allusion à un évènement non exclusivement discursif, la loi encadrant le droit de manifester. Or, ce type d'évènement fait néanmoins l'objet de discours. Ici, c'est 
par la proposition d'un texte de loi que cette dernière est promulguée. Il en est de même d'autres évènements en apparence non discursifs: un conflit armé est précédé généralement, à tout le moins - de pourparlers et d'une déclaration officielle de guerre, un évènement politique comme un référendum fait l'objet, avant, pendant et après, de nombreux discours, etc. Tout fait historique ou presque se construit donc en discours. Les catastrophes naturelles sont peut-être les seuls évènements qui ne proviennent pas de discours, mais leur venue en provoque toujours - et il en est de même de leur éventualité ; pensons seulement aux discours sur la prévention du risque -, et ce sont ces discours, en partie du moins, qui participent à rendre l'évènement mémorable et à l'inscrire dans l'histoire. Dans le cas qui nous intéresse ici, c'est le désaccord et le dissensus autour d'une loi jugée restrictive et perçue comme une atteinte à la liberté de manifester qui constitue le « déjà-là » à partir duquel des slogans sont construits.

Parmi les autres slogans appartenant à cette catégorie, un est particulièrement révélateur des mécanismes de circulation du discours. Pour bien le comprendre, il est toutefois nécessaire de retourner au discours source et de préciser le contexte dans lequel il a été produit. Le 20 avril 2012, alors que le conflit étudiant bat son plein, le premier ministre J. Charest prononce un discours au salon du Plan Nord ${ }^{4}$, pendant que des centaines de personnes manifestent à l'extérieur du Palais des congrès de Montréal, où se tient l'évènement. Pendant son discours d'ouverture, et en référence aux manifestants en question, il tient les propos suivants :

Le salon du Plan Nord que nous allons ouvrir aujourd'hui qui est déjà très populaire, les gens courent de partout pour entrer [rires et applaudissements du public]... est une occasion [sourire de J. Charest]... une occasion notamment pour les chercheurs d'emploi [rires de J. Charest et du public, applaudissements du public]... alors à ceux qui frappaient à notre porte ce matin on pourra leur offrir un emploi [rires du public, sourire de J. Charest] dans le Nord autant que possible [rires de J. Charest et du public, applaudissements du public].

Les propos de J. Charest sont rapidement médiatisés : d'une part, le salon du Plan Nord est attendu depuis longtemps et, en lui-même, le Plan Nord est très controversé et fait la manchette depuis déjà plusieurs mois ; d'autre part, avril 2012 marque le cœur de la grève étudiante, et les manifestations se font de plus en plus nombreuses et des affrontements virulents, et parfois violents, sont rapportés tous les jours dans les médias. Il n'est donc pas étonnant, dans ce contexte, que les propos de J. Charest soient largement diffusés. C'est suivant l'allocution de J. Charest que le slogan suivant, tant scandé qu'écrit, apparait dans les manifestations étudiantes: «Charest, dehors, on va te trouver une job ${ }^{5}$ dans le Nord ».

Il y a transformation du discours source puisque les manifestants ne reprennent pas strictement les propos de J. Charest, mais ils y font clairement allusion afin de les décrier ${ }^{6}$ , le tout en faisant pression sur autrui. En effet, " on va te trouver une job dans le Nord" constitue une forme de menace : on va te faire perdre ton poste de Premier ministre. Un tel acte de discours "projette, sous forme injonctive, un désir de domination" (Fracchiolla \& Moïse, 2009, p. 120). Évidemment, les manifestants savent que la teneur de cette menace est non exécutoire puisqu'ils n'ont pas le pouvoir d'envoyer J. Charest travailler dans le nord de la province. Néanmoins, ils sont conscients de leur rôle dans la vie démocratique et savent qu'éventuellement, un appel au vote contre J. Charest et son parti peut porter ses fruits. Ainsi, toujours selon B. Fracchiolla et C. Moïse (ibid.), «la plupart des menaces [proférées en période d'affrontement social] visent directement le rôle institutionnel des hommes politiques au cœur de la critique - s'ils persévèrent dans 
leurs erreurs et dans leurs volontés, ici contraires à celles du peuple, ils ne seront pas réélus ».

La reprise est effacée puisqu'il n'y a aucune marque linguistique de discours rapporté et qu'il s'agit surtout de jeter un regard (négatif) sur J. Charest et sur sa politique davantage que sur son discours lui-même. Ce phénomène est fréquent dans les slogans revendicateurs, qui participent entre autres à créer des images types de chaque protagoniste tenu pour responsable de la crise. En faisant référence au discours controversé du premier ministre, les manifestants vont donc au-delà de ses propos et s'attaquent à sa personne ${ }^{7}$, de même qu'à son attitude dans le conflit étudiant, dont le discours source témoigne - blaguer sur un conflit qui perdure a été décrié par plusieurs, tant chez les pro- que chez les antiétudiants. En détournant le discours de J. Charest, en se positionnant contre lui et en faisant pression sur son énonciateur, les manifestants appuient leur point de vue tout en jouant avec un fait d'actualité, ce qui en a amené plusieurs à souligner cette originalité.

\section{Le rappel de références culturelles}

Enfin, sur le plan quantitatif, la catégorie la plus importante du corpus est la remise en discours de références culturelles, quelle qu'en soit la nature. Cette catégorie s'apparente à la première, soit la reprise de slogans revendicateurs classiques, en ce que ce sont des paroles souvent très connues qui sont reprises. Or, à l'inverse, le contenu des discours rapportés, ici, ne s'inscrit pas de prime abord dans un contexte de protestation.

Parmi ces slogans, notons les nombreux «Je pense, donc je nuis » (sur le modèle de « Je pense, donc je suis » de R. Descartes), «Ah! comme la hausse a haussé » (transformation de " Ah! comme la neige a neigé », vers d'É. Nelligan) et " Nous sommes arrivés à ce qui commence » (de "Je suis arrivé à ce qui commence ", tiré d'un poème de G. Miron). Ces trois slogans mettent en œuvre les mêmes phénomènes : une légère transformation du discours source dont la reprise est effacée, c'est-à-dire qu'elle n'est pas accompagnée de marques de discours rapporté, tantôt pour l'inscrire au cœur du mouvement étudiant ( $\mathrm{Ah}$ ! comme la hausse a haussé », qui rappelle l'importante augmentation des frais de scolarité universitaires), tantôt pour donner au mouvement une impression de grandeur (« Nous sommes arrivés à ce qui commence ») et pour permettre aux manifestants de construire un ethos collectif (Orkibi, 2008) contestataire et socialement actif ( $«$ Je pense, donc je nuis»). Notons en outre que "Nous sommes arrivés à ce qui commence", davantage qu'une reprise directe du discours source, constitue plutôt la reprise d'une reprise : en effet, cette appropriation à la première personne du pluriel du vers de G. Miron a servi de conclusion à Nous?, un évènement de prise de parole publique sur le Québec et la démocratie qui a eu lieu en avril 2012, évènement auquel les leaders des associations étudiantes à la tête de la grève ont participé, et où l'un d'entre eux a lui aussi repris les paroles du poète. C'est à la suite de cet évènement que le slogan s'est fait lire (et entendre) dans les manifestations étudiantes. On peut alors se demander qui est perçu comme l'énonciateur du discours source par les individus qui le remettent en discours en le transformant, G. Miron ou les organisateurs de l'évènement Nous? Sans pouvoir répondre avec certitude à cette question, nous avançons que c'est en écho à cet évènement que les manifestants brandissent " Nous sommes arrivés à ce qui commence " puisque c'est à sa suite qu'on voit apparaitre cette phrase, identique à celle prononcée à ce moment, sur des pancartes revendicatrices et que les propos tenus par les leaders 
étudiants sont plus frais dans la mémoire des manifestants que le poème de G. Miron. De là, on peut observer le caractère multiple du discours en circulation, qui est repris, transformé et réutilisé suivant divers maillons de la chaine.

21 Ces slogans, construits à partir de références que l'on peut dire savantes ou lettrées dans l'ensemble du corpus, comme dans les exemples précédents, ce sont les reprises d'extraits d'œuvres ou d'ouvrages littéraires, philosophiques, historiques ou politiques qui dominent - participent à l'image d'un groupe qui revendique à raison. En effet, le recours à des discours typiquement appris ou analysés en contexte scolaire est cohérent avec les demandes d'un groupe qui manifeste pour un accès non limité à l'éducation. Les manifestants construisent alors l'image d'un groupe instruit, cultivé et politisé.

22 À l'inverse, bon nombre de références populaires - et souvent caractéristiques de la génération à laquelle appartiennent la plupart des manifestants - sont aussi réinvesties sous forme de slogans. L'effet est alors moins la mise en avant d'un ethos "savant" servant notamment à véhiculer une image positive du groupe à l'extérieur, mais plutôt la construction, au sein même du groupe, d'une identité fondée sur des références communes largement (re)connues. C'est dans ce contexte qu'apparait « Passe-Montagne aime les papillons, les souliers neufs et l'éducation ", transformation d'une portion de la chanson thème d'une émission pour enfants très populaire au Québec dans les années 80 , Passe-Partout. L'original se présentait comme suit : «Passe-Montagne [un personnage de l'émission] aime les papillons, les souliers neufs et les beaux vestons ». Ici, c'est la rime entre vestons et éducation qui sert de base à la transformation du discours source. L'imaginaire collectif enfantin est alors mobilisé, comme c'est également le cas dans « Poudlard, c'est gratuit!», référence directe à la saga Harry Potter servant à prôner non seulement l'accès à l'éducation, mais plus encore, la gratuité scolaire. Sensiblement dans la même veine, mais sans référence directe aux revendications étudiantes, on note plusieurs variations sur le thème de "Line la pas fine $e^{8}$ ", qui est le surnom d'un personnage féminin de la série télévisée Les invincibles et qui, ici, est aussi employé pour désigner la ministre de l'Éducation, Line Beauchamp. Dans les trois cas présentés, la référence à partir de laquelle les slogans sont formés est connue à un point tel qu'elle ne nécessite aucune explication, ni aucune marque de reprise d'ailleurs: l'ensemble des manifestants - comme l'ensemble de la société québécoise, d'ailleurs - comprend sans même réfléchir ce à quoi réfèrent Poudlard, Passe-Montagne et Line la pas fine. L'utilisation de ces références culturelles comme base de discours de l'appel collectif permet de donner un aspect ludique et plus léger à des revendications dont la teneur, elle, ne l'est pas.

\section{Conclusion}

23 Les discours circulent sans cesse, et ce, de différentes façons. Qu'il s'agisse de discours rapporté sous sa forme la plus classique, de reformulation, d'allusion ou de réappropriation, un élément est commun à tous ces phénomènes : un discours source est repris par un tiers, souvent dans une optique de positionnement idéologique. Les slogans du printemps érable n'y font pas exception, et on constate que bon nombre sont construits par la remise en discours de propos sources, que ces derniers soient repris fidèlement ou non. Trois types de reprise sont observables dans le corpus, et on peut alors les considérer comme des prototypes de la circulation des discours réinvestis pour former des slogans revendicateurs. 
24 Il n'est pas étonnant que plusieurs slogans proviennent de la reprise de discours manifestants préalablement énoncés, parfois même de façon si fréquente qu'ils constituent des incontournables de la parole contestataire, comme si un mouvement en appelait un autre, qui se nourrissaient mutuellement et qui, à leur tour, allaient nourrir les prochains épisodes de même nature. Dans un tel cas, les discours sources sont soit repris tels quels, soit légèrement transformés, et les "nouveaux» slogans suivent toujours une tonalité euphorique et une orientation convergente. Ici, c'est l'inscription d'un discours manifestant dans un contexte de production plus large, mais quasiidentique au sien, qui doit être soulignée et qui participe à la mobilisation du groupe.

À l'inverse, le détournement et la réappropriation du discours et des idées de l'opposant servent à prendre ses distances de façon marquée de ce dernier, à le critiquer et à en véhiculer une image négative. Reprendre son discours et en faire la base de slogans revendicateurs ne signifie en aucun cas une adhésion aux idées de l'adversaire. Au contraire, par des allusions tantôt ironiques, tantôt explicitement critiquées, donc par une antiorientation avec les propos sources, les manifestants se servent de ces derniers pour condamner le gouvernement et ses prises de position.

Enfin, la reprise et l'allusion à des références culturelles, tant savantes que populaires, permettent aux manifestants de se détacher - dans une certaine mesure - du strict affrontement auquel ils participent pour faire appel à des connaissances générales partagées par plusieurs. Ce faisant, ils font montre autant d'une identité « lettrée » qui va de pair avec leurs revendications concernant l'accès à l'éducation supérieure que d'une identité de groupe partageant des références communes. L'objectif est alors de réinvestir des éléments culturels qui ne concernent initialement en rien la grève étudiante et de les remobiliser dans un tel contexte.

En somme, on constate le rôle et l'importance de la réactualisation et de la remise en circulation de discours du passé - proche ou lointain - pour appuyer son point de vue. De par les slogans scripturaux qu'ils ont mis de l'avant, les individus ayant participé aux manifestations entourant la grève étudiante québécoise de 2012 ont témoigné de la place qu'occupe le "déjà-là » dans la construction de leurs discours revendicateurs: un matériau discursif à réinvestir, à remettre en circulation, à se réapproprier et à transformer dans une optique d'appel à l'action collective.

\section{BIBLIOGRAPHIE}

AUthieR-ReVUZ, J. (2000). « Aux risques de l'allusion ». In : Murat, M. (dir.) L'allusion dans la littérature. Paris : Presses universitaires de la Sorbonne, p. 209-235.

Bakhtine, M. (1977) [1929]. Le marxisme et la philosophie du langage. Essai d'application de la méthode sociologique en linguistique. Paris : Éditions de Minuit.

BERNARD BARBEAU, G. (2015). « De l'appel à la mobilisation à ses mécanismes sociodiscursifs : le cas des slogans revendicateurs écrits du printemps érable ». Argumentation et analyse du discours 14. En ligne : http://aad.revues.org/1969. 
BONENFANT, M., GLINOER, A. \& LAPOINTE, M.-E. (2013). Le printemps québécois : une anthologie. Montréal : Les Éditions Écosociété.

DENTON, R. (1980). « The rhetorical functions of slogans: Classifications and characteristics ». Communication Quarterly 28, p. 10-18.

FRACCHIOLLA, B. \& MOÏSE, C. (2009). « Construction de la violence verbale et circulation des discours : autour du contrat de première embauche ». In : López Muñoz, J. M., Marnette, S., Rosier, L. \& Vincent, D. (dirs). La circulation des discours. Québec : Nota bene, p. 103-125.

GRINSHPUN, Y. (2013). « Discours manifestant et contestation universitaire (2009) ». Argumentation et analyse du discours 10. En ligne : http://aad.revues.org/1476.

LAFOREST, M. \& MOÏSE, C. (2013). « Entre reproche et insulte, comment définir les actes de condamnation? » In : Fracchiolla, B., Moïse, C., Romain, C. \& Auger, N. (dirs). Violences verbales. Analyses, enjeux et perspectives. Rennes: Presses universitaires de Rennes, p. 85-101.

LÓPEZ MUÑOZ, J. M., MARNETTE, S. \& ROSIER, L. (dir.) (2004). Le discours rapporté dans tous ses états. Paris : L'Harmattan.

ORKIBI, E. (2008). «Ethos collectif et Rhétorique de polarisation : le discours des étudiants en France pendant la guerre d'Algérie ». Argumentation et analyse du discours 1. En ligne $: \underline{\text { http:// }}$ aad.revues.org/438.

REBOUL, O. (1975). Le slogan. Bruxelles : Complexe.

ROSIER, L. (2003). « Du discours rapporté à la circulation du discours : l'exemple des dictionnaires de "critique ironique" ». Estudios de Lengua y Literatura francesas 14, p. 63-82.

TURBIDE, O., VINCENT, D. \& LAFOREST, M. (2010). « The circulation of discourse: The case of deprecating remarks on trash radio ». Discourse Studies 12. 6, p. 785-801.

VINCENT D., LAFOREST, M. \& TURBIDE, O. (2007). « Une boîte de Pandore : de l'analyse de discours radiophoniques à l'intervention sociale ». Communications 25. 2, p. 187-199.

VINCENT, N. (2014). «Créativité et identité dans la langue de la contestation : le cas du printemps érable ». In : Romain, C. (dir.), Les locuteurs et les langues : pouvoirs, non-pouvoirs, contre-pouvoirs. Limoges : Lambert Lucas, p. 331-342.

\section{NOTES}

1. Ce qui correspond environ à $1200 €$.

2. Pour une présentation complète et une chronologie détaillée des évènements, nous renvoyons à M. Bonenfant, A. Glinoer et M.-E. Lapointe (2013).

3. Nous remercions Marie Barré pour son aide à la constitution du corpus.

4. Le Plan Nord est un programme de développement économique des régions nordiques du Québec qui a été vivement critiqué dans la population.

5. Job est employé au féminin au Québec.

6. J. Authier-Revuz (2000) souligne à juste titre que pour que l'allusion remplisse son rôle, elle doit être comprise du récepteur. Ici, les manifestants courent certes le risque qu'autrui ne voie pas le lien entre le slogan et les propos de J. Charest, mais ce risque est amoindri par la vaste diffusion du discours de J. Charest. Par ailleurs, le risque peut valoir la chandelle puisqu'un effet positif est possible, celui de la cohésion sociale : se crée en effet un groupe d'initiés qui comprend l'allusion et qui, plus encore, se l'approprie. 
7. Sur le glissement des condamnations du faire (qui portent sur des actions) aux condamnations de l'être (qui visent un individu), voir M. Laforest et C. Moïse (2013).

8. Au Québec, être fin signifie être sympathique, être gentil. Une personne "pas fine " est mesquine, méchante.

\section{RÉSUMÉS}

En 2012, le Québec a connu la plus importante grève étudiante de son histoire. Parmi la production discursive découlant de ce vaste mouvement de contestation, les slogans entendus ou vus lors des manifestations ont suscité l'intérêt dans l'espace public. C'est à cet objet, et plus particulièrement au slogan revendicateur sous sa forme scripturale, que cet article est consacré. Nous nous intéressons plus spécifiquement à la façon dont les manifestants ont recours au discours d'autrui pour construire leurs propres énoncés revendicateurs. Nous inscrivant dans une approche sociodiscursive de l'analyse de la circulation des discours, nous nous proposons d'étudier la reprise, la réinterprétation et la remise en discours des propos d'autrui dans les slogans scripturaux du printemps érable afin de faire ressortir la manière dont les individus réactualisent et remettent en circulation des discours du passé - proche ou lointain - pour appuyer leur point de vue.

In 2012, Quebec experienced the largest student strike in its history. Among the discursive production issuing from this protest movement, slogans both chanted and written have generated interest in the public space. This article focuses on the latter. Following a sociodiscursive approach to the circulation of discourse, our aim is to examine the ways through which do protesters repeat, reinterpret and reformulate past discourses in order to build their own slogans and articulate their point of view.

\section{INDEX}

Mots-clés : circulation des discours, slogans, revendication, Québec

Keywords : circulation of discourse, slogans, protest, Quebec

\section{AUTEUR \\ GENEVIÈVE BERNARD BARBEAU}

Université du Québec à Trois-Rivières, Canada 